\title{
Research on the development method of course arranging management software based on UML
}

\author{
Guoliang Wang $^{1, \mathrm{a}}$, Xiaoming Huang ${ }^{2, \mathrm{~b}}$ \\ e-mail: ${ }^{1, a} 12931482 @ q q . c o m ;{ }^{2, b} 651769635 @ q q . c o m$ \\ Nanchang institute of science \& technology, China
}

Keywords: software development, UML, RRUP, courses arranging management system

\begin{abstract}
Courses arranging management system is designed and developed to solve the school complex courses scheduling, its basic requirement is to realize the semi-automatic or automatic course arrangement, the courses arranged must be reasonable and practical. This article combines RRUP process to introduce each model which is represented by UML in the actual development of various courses arranging management system.

We use the use case model to represent the system model of the requirements phase, the use case model is mainly made up of use case diagram, from this system to the final release of subsystem, each iteration of its use case model is not the same. In the development process of courses arrangement system, along with the continuous renewal of iteration, the use case model is constantly changing. Due to the limitation of space, this paper only presented the use case model which determined by the first iteration and last iteration.

Through the analysis of requirements, determined the functional requirements of courses arrangement system, its requirements are briefly summarized as follows:

(1)Courses arranging information settings: including course information, class time, limited information of subjects and teachers, class arrangement information, courses arranging management system according to these courses arranging information and limited information to automatically arrange the courses.
\end{abstract}

(2)Manual and automatic courses arrangement: after the user set the courses arrangement information, the system can automatically arrange the class schedule, also can manually adjust the arranged schedule. In the process of course arrangement, it can prompt the users for the unreasonable result of courses arrangement.

(3)Schedule report and schedule inquiry: give the entire school teachers and class schedules; the user can select different teachers, classes, subjects in schedule inquiry, system according to different users to choose the corresponding schedules.

Requirements describe is the beginning of the whole system in the primary stage, disapprove the user to describe the requirements using document in RRUP, but use the use case diagram and use case model to establish the whole demand model for the system.

At the beginning of the project, the demand is not very clear, however, its demand center content is still above points, through the analysis of demand, we identified a few very important use cases: subject information setting, class arrangement information setting, automatic course arrangement, schedule adjustment, schedule display and print.

The use cases listed above are the major cases of arrangement system. According to these use cases, in the primary stage of the project, determined the initial use case model for the arrangement 
system, described the function of courses arrangement system should be completed, that is, from the user's point of view, what functions that the system should have. In the first iterative development of project development, it is based on the above determined system prototype, which determined the initial structure of system arrangement. In iterative development, which after the courses arranging management system, is extended on the basis of this model.

Figure 1 Courses arranging management use case diagram

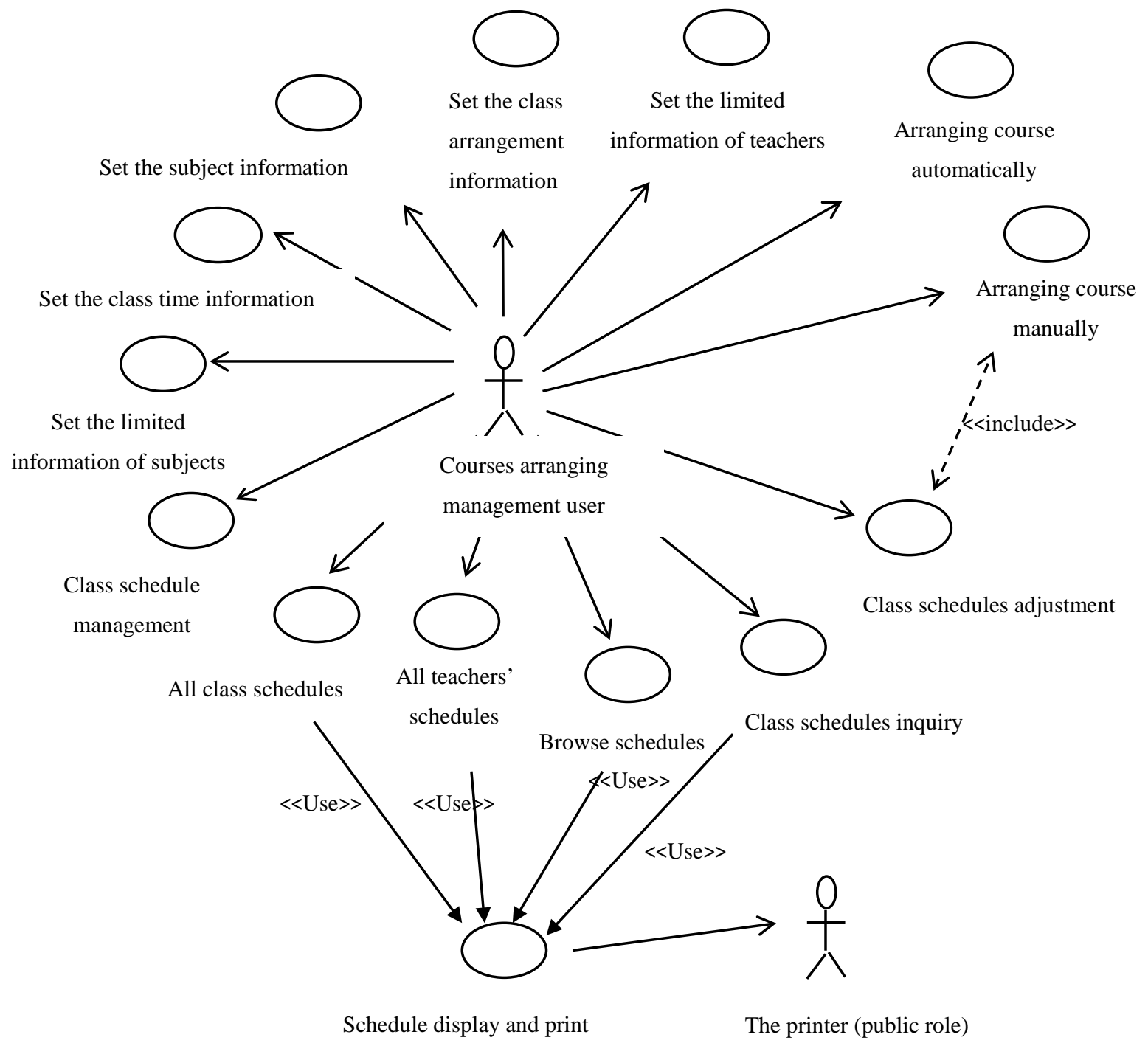

Through several iterations, under the new demand growth and the further understanding of the system, gradually perfect the use case model of courses arrangement system. The use case diagram of courses arranging management includes all roles and use cases of courses arrangement system, in the actual UML models, multiple use case diagrams are merged into one diagram. As shown in figure 1.

Here the use case model is given in the current iteration, does not represent the model is most complete and optimal, the model will be continuously optimized and improved with the deepening of the iterative development.

The use case model of system was given above, for each use case on use case diagram, just gave the name of use cases, and did not give the description and explanation of use case. When modeling, we must give the instructions of each use case, describe the complete function of the use 
case and steps to complete the function of the use case. In the description of use case, I chose the UML activity diagram function, of course, the description of the use case can also be represented by the use cases documentation. To illustrate how to use activity diagrams to represent the behavior of a use case, gave the "automatic course arrangement" use case activity diagram, as shown in figure 2.

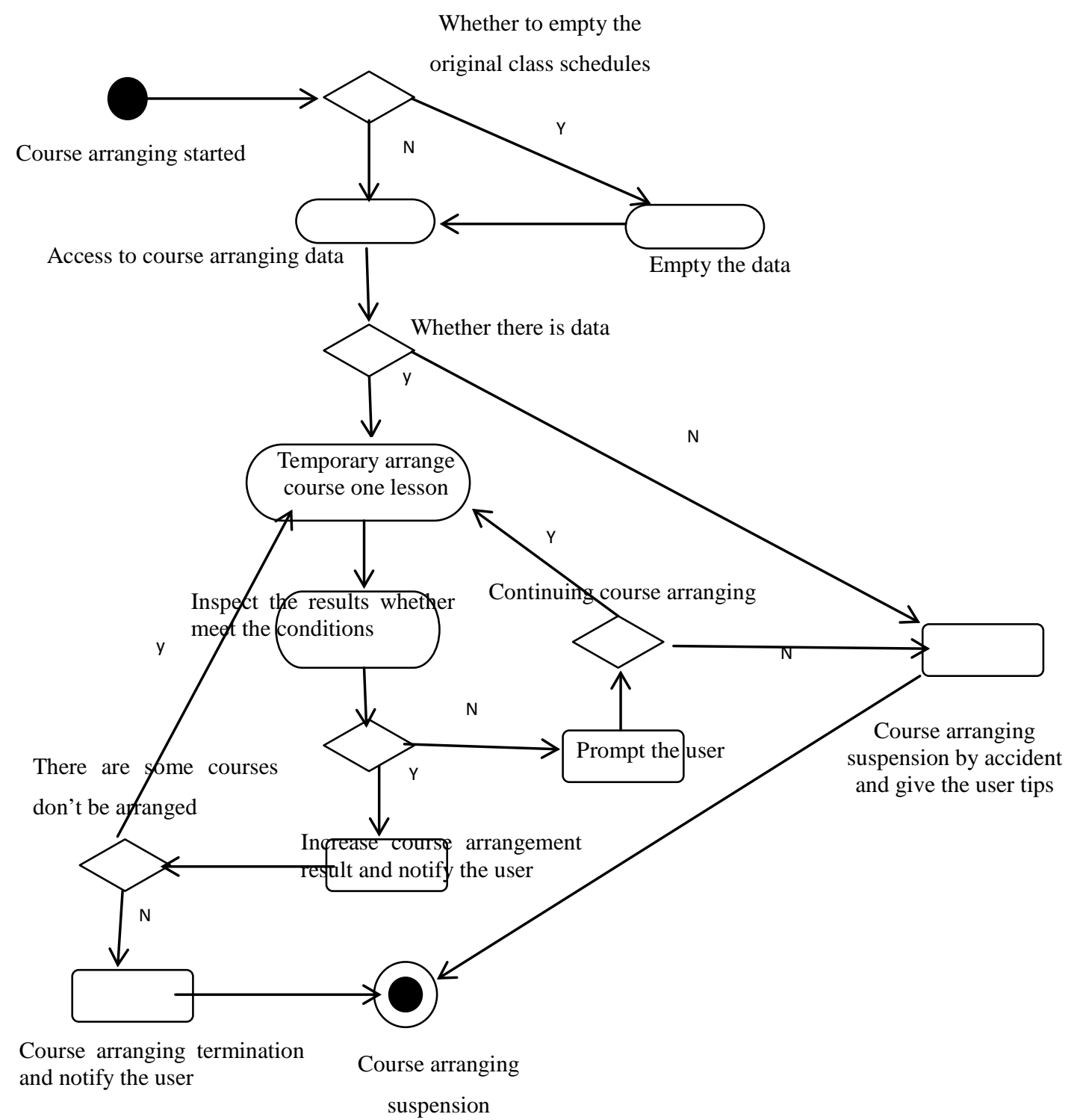

Figure 2 Automatic course arrangement activity diagram

The activity diagram above in detail described the automatic arrangement use case in the actual execution time, what steps it should have, including his successful implementation of the user's expectations and steps that are not successfully performed. In the steps of using the activity diagram to describe the course use cases, some activities may need be optimized, including increased some activities or merge some steps, all of these will be optimized with the continuous development of iterative development.

Analysis model is created in the development of the whole project is crucial, because this is a stage that will use the model to be transformed into the category that should exist in the system, is 
the stage that how to implement system function with category. The back of the whole project development work, it is carried out on the basis of analysis model for analyzing the section of the complete, so in the process of the project development, should ensure the quality of the work in this phase, strictly complete all kinds of UML diagrams in this phase.

At this stage, find out the main category involved in the courses arranging management system, and combine with the use case of the use case model, organically combine with the category and use cases, establish the corresponding category diagrams to represent the relationship between the categories. And how to make the use case with the union, you need to set up a corresponding sequence diagram/ assistance figure.

Analyzing the establishment of model, not one or a few category diagram can achieve, in order to adequately model for one system, for different projects can choose different modeling elements and modeling mechanism. In modeling of arrangement system, chose the category diagram and sequence diagram to build the corresponding analysis model.

UML is a standard modeling language in the new period of software development, this paper introduced the UML, analyzed the various modeling elements and modeling mechanism of UML, and pointed out the model elements and modeling mechanism should be used in what situation, it has a certain guiding significance for modeling software developers work .

\section{References}

[1]Chunhui Jiang. The design and implementation of the newspaper sales management system based on UML [D]. Zhengzhou: Zhengzhou University, 2010.

[2]Shun Na. The design and implementation of the educational administration management system for adult education [J]. National higher education research, the 5 phase of 2011.

[3]Fushun Li. The development of educational administration information system project [D]. East China Normal University, 2010.

[4]Yinghua Tong. Analysis and design of educational administration system in colleges and universities [D]. Shandong: Tongji University, 2006.

[5]Ming Sun. The application and analysis of educational administration management system in colleges and universities based on asp.net [J]. Technology information, the 26 phase of 2010.

[6]Jianhua Liu. Demand management tool based on BS structure -- Research on business modeling [D]. North China University of Technology, 2004. 